\title{
CONTAMINACIÓN PRODUCIDA DURANTE EL PROCESADO, PREPARACIÓN, TRANSPORTEY LIMPIEZA DE LOS ALIMENTOS
}

\author{
DRA. PILAR FERNÁNDEZ HERNANDO \\ Departamento de Ciencias Analíticas. \\ Facultad de Ciencias. UNED
}

\section{CONTAMINACIÓN PRODUCIDA DURANTE EL PROCESADOY PREPARACIÓN DE LOS ALIMENTOS}

Los alimentos que consumimos hoy en día pueden tener diversas sustancias químicas, como resultado de su uso directo o como contaminantes inevitables procedentes del medio ambiente o del procesado. Muchas sustancias químicas juegan un papel esencial en la producción y distribución de los alimentos. Así, en las primeras etapas de la producción se aplican plaguicidas a los productos vegetales para combatir plagas y se administran medicamentos veterinarios a los animales de granja para luchar contra las enfermedades.

En etapas posteriores, durante el procesado y la preparación de alimentos, podríamos decir que las reacciones químicas son probablemente infinitas. Entre las diferentes contaminaciones que se pueden producir, destacan las debidas al uso de aditivos alimentarios y a los envoltorios o embalajes que, en general, contribuyen a mantener la higiene de los alimentos y a hacerlos más atractivos. El uso de estas sustancias químicas aporta claros beneficios y ventajas, pero siempre hay que tener en cuenta el potencial riesgo para la salud del consumidor derivado de los residuos que quedan de ellas en los alimentos.

En general, un tóxico generado durante el procesado es parte intrínseca de las transformaciones de un alimento.

\section{Tratamientos químicos: Aditivos Alimentarios}

En esta parte del curso vamos a mostrar los diferentes productos que se 
añaden a los alimentos para mejorar sus propiedades o su conservación, y otros que se usan para facilitar los procesos de fabricación, aunque no permanecen en el producto final.

\section{Un aditivo se puede definir como:}

Una sustancia o mezcla de sustancias diferentes al alimento, que se encuentran en el mismo, como resultado de producción, almacenamiento o empacado, añadido intencionalmente para lograr ciertos beneficios, como mejorar el nivel nutritivo, conservar la frescura, impedir el deterioro por microorganismos e insectos, generar alguna propiedad sensorial deseable o bien como ayuda de proceso.

El empleo de aditivos en alimentos se viene utilizando desde tiempos remotos. Existen datos que prueban el uso de la sal y el ahumado desde tiempos prehistóricos. Los romanos utilizaban el salitre y los derivados de azufre como conservantes. La práctica de colorear los alimentos también tiene una larga tradición, ya que algunos productos naturales como el azafrán o la cochinilla (ácido carmínico) eran ya conocidos por las civilizaciones antiguas, su conocimiento se remonta a la civilización egipcia y romana, cuyos reyes y emperadores ya los utilizaban.

Los colorantes se empleaban y se siguen empleando, mayoritariamente, para dar un mejor aspecto a los alimentos. No obstante, el tipo de colorantes empleados ha cambiado considerablemente en el transcurso de los años.

Otro tipo de aditivos destacados son los empleados en la conservación de los alimentos. Algunos de los métodos de estabilización y conservación, así como los aditivos añadidos a los alimentos, son utilizados hoy en día sin apenas diferencias con los utilizados por nuestros antepasados, por ejemplo los métodos de salazón y secado de pescado utilizados en las zonas costeras mediterránea, son prácticamente idénticos a los utilizados por los fenicios, de hecho son herencia de ellos. Los lácteos fermentados, quesos, jamones, embutidos curados, encurtidos, entre otros, son alimentos en los que se han utilizado procedimientos de conservación, para los que el ser humano se valió de conocimientos empíricos en su elaboración.

El cambio en los modos de vida experimentado en las últimas décadas, pasando de una sociedad agraria con un alto grado de autoabastecimiento a una más industrializada, urbana y especializada, junto con el desarrollo de la tecnología y la exigencia por parte del consumidor de alimentos de más calidad y seguridad, hace indispensable hoy en día la utilización de aditivos por parte de la industria alimentaria. 
Si bien es cierto que el empleo de aditivos de forma descontrolada podrá ocasionar algún problema sanitario y de salud pública, no es menos cierto que gran parte de los productos alimenticios que consumimos hoy en día no podrían existir sin el empleo de estos compuestos.

El uso de aditivos alimentarios junto con las nuevas tecnologías ha posibilitado la aparición de nuevos productos adaptados a las exigencias de la vida actual tales como platos preparados, postres instantáneos, panes de molde, margarinas hidrogenadas, alimentos tipo fast-food, aperitivos o snacks, salsas, condimentos y otros.

\section{Por qué se añaden los aditivos a los alimentos}

Es difícil demostrar que un aditivo presenta algún beneficio al alimento, salvo determinados conservantes. A continuación, se muestran algunas razones más importantes por las que se añaden los aditivos a los alimentos:

Valor nutritivo: Para aumentar el valor nutritivo se añaden vitaminas, minerales, aminoácidos o sus derivados.

Valor organoléptico o sensorial: Color, olor, sabor y consistencia o textura son caracteres importantes para determinar la calidad sensorial de un alimento, y todos ellos pueden verse alterados por el procesado o almacenamiento. Dichos cambios se compensan con la adición de colorantes, sustancias aromáticas o potenciadores del sabor ("flavour enhancer"). El desarrollo de aromas no deseados ("off flavour"), debido por ejemplo al enranciarniento de las grasas, se impide con antioxidantes. La consistencia se estabiliza en muchos casos con la adición de polisacáridos, minerales y de otras muchas formas.

Vida útil: La conservación de los alimentos afecta tanto a la prevención de las alteraciones de origen microbiano, como a la detención y retraso de modificaciones químicas y físicas indeseables, estabilizando por ejemplo el pH con ayuda de espesantes o gelíficantes de tipo polisacárido.

Comodidad: La tendencia creciente hacia alimentos de preparación rápida y sencilla (“convenience food”) condiciona también un uso mayor de aditivos.

\section{Control y legislación de los aditivos}

Todos los aditivos alimentarios deben tener un propósito útil demostrado 
y han de someterse a una valoración científica rigurosa y completa para garantizar su seguridad, antes de que se autorice su uso. El comité que se encarga de evaluar la seguridad de los aditivos en Europa es el Comité Científico para la Alimentación Humana de la UE (Scientific Committee for Food, SCF). Además a nivel internacional, hay un Comité Conjunto de Expertos en Aditivos Alimentarios (Joint Expert Committeee on Aditivos alimentarios, JECFA) que trabaja bajo los auspicios de la Organización de las Naciones Unidas para la Agricultura y la Alimentación (FAO), y la Organización Mundial de la Salud (OMS).

Sus valoraciones se basan en la revisión de todos los datos toxicológicos disponibles, incluidos los resultados de las pruebas efectuadas en humanos y animales. A partir del análisis de los datos de los que disponen, se determina un nivel dietético máximo del aditivo, que no tenga efectos tóxicos demostrables. Dicho contenido es denominado el «nivel sin efecto adverso observado» («noobserved-adverse-effect level» o (NOAEL) y se emplea para determinar la cantidad de «ingesta diaria admisible» (IDA) para cada aditivo. La IDA, que se calcula con un amplio margen de seguridad, es la cantidad de un aditivo alimentario que puede ser consumida en la dieta diariamente, durante toda la vida, sin que represente un riesgo para la salud.

\section{Código E y Código $H$}

Todos los aditivos alimentarios se representan con un código, que empieza por la letra E y sigue con un número. Estos aditivos están legalizados en todos los países de la Unión Europea.

Para todos los aditivos se sigue ese sistema de codificación de la E y los números, de modo que los colorantes figuran entre el E 100 y e1 190.

Los conservantes son todos aquellos que en las etiquetas de productos alimentarios figuran entre la E 200 que es el ácido sórbico y la E 290 del anhídrido carbónico. Los antioxidantes se reconocen porque van de la E 300 del ácido ascórbico al E 385 del atilenodiamino tetracetato cálcico disódico. Por su parte, los estabilizantes, espesantes, emulgentes y gelificantes van del E 400 que es el ácido algínico (polisacárido coloidal) al E 585 que es el lactato ferroso. Los potenciadores de sabor comienzan con el ácido glutámico, cuyo código es el E 620, y finalizan con el dimetilpolisiloxano, que es el E 900. Los agentes de recubrimiento van del E 901 al E 914. Los gases se identifican por los códigos que van del E 938 del argón, al E 948 del oxígeno. Con el E 950 comienzan los edulcorantes, que finalizan con el E 967. 


\section{Código $\boldsymbol{H}$}

Se codifican con la letra $\mathrm{H}$ más cuatro cifras los aditivos admitidos en España pero no autorizados en todos los países europeos.

\section{Clasificación de los aditivos}

Originalmente los aditivos fueron clasificados por su origen en naturales y sintéticos. Esta clasificación aunque lógica contribuyó durante algún tiempo al mantenimiento de una dualidad errónea en la que se equiparaba a lo natural con lo sano y a lo sintético con lo peligroso y que podía colocar al consumidor en una actitud equivocada.

Una buena clasificación en función de los beneficios y usos: sensoriales, estéticos y cosméticos se muestra en la tabla 1.

Tabla 1: Clasificación de los aditivos en función de los beneficios y usos: sensoriales, estéticos y cosméticos.

\begin{tabular}{|c|c|c|c|c|}
\hline SABOR/OLOR & APARIENCIA & ESTABILIDAD & TEXTURA & EMPAQUE \\
\hline $\begin{array}{c}\text { Aroma } \\
\text { Sabor } \\
\text { - dulce } \\
\text { - ácido } \\
\text { - salado } \\
\text { - amargo }\end{array}$ & $\begin{array}{l}\text { Color } \\
\text { Claridad } \\
\text { Opacidad } \\
\text { Espuma } \\
\text { Turbidez }\end{array}$ & $\begin{array}{c}\text { Conservantes } \\
\text { Antioxidantes } \\
\text { Emulsionantes } \\
\text { Dispersantes }\end{array}$ & $\begin{array}{l}\text { Firmeza } \\
\text { Seco } \\
\text { Polvoriento } \\
\text { Masticable } \\
\text { Retención } \\
\text { Humedad } \\
\text { Fracturable }\end{array}$ & $\begin{array}{c}\text { Sanidad } \\
\text { Etiqueta } \\
\text { Conservación } \\
\text { Atracción } \\
\text { Estabilidad }\end{array}$ \\
\hline $\begin{array}{l}\text { Sensaciones } \\
\text { - pungente } \\
\text { - frescura }\end{array}$ & & & & \\
\hline
\end{tabular}

Otra clasificación, mucho más amplia, podría hacerse en función de los aditivos que mejoran las propiedades organolépticas o las que impiden las alteraciones o los que mejoran su textura. Según esta clasificación como se muestra en la tabla 2. 
Tabla 2: Clasificación general de aditivos en función de su influencia en las propiedades organolépticas.

\begin{tabular}{|c|c|c|}
\hline & Edulcorantes & $\begin{array}{l}\text { Sacarina. Aspartame. } \\
\text { Acesulfame. Hidrochalconas. }\end{array}$ \\
\hline \multirow{5}{*}{$\begin{array}{l}\text { Aditivos que } \\
\text { mejoran las } \\
\text { propiedades } \\
\text { organolépticas }\end{array}$} & $\begin{array}{l}\text { Aromatizantes y } \\
\text { saborizantes }\end{array}$ & $\begin{array}{l}\text { Extractos naturales aromáticos. } \\
\text { Aromas destilados. Aromas sintéticos. } \\
\text { Glutamato monosódico. } \\
\text { Inosin-mononucleótido. } \\
\text { Guanin-mononucleótido. Maltol }\end{array}$ \\
\hline & $\begin{array}{l}\text { Colorantes } \\
\text { naturales }\end{array}$ & $\begin{array}{l}\text { Clorofila. } \\
\text { Carotenoides obtenidos de plantas. } \\
\text { Anfocianos. } \\
\text { Carmín. }\end{array}$ \\
\hline & $\begin{array}{l}\text { Colorantes } \\
\text { sintéticos }\end{array}$ & $\begin{array}{l}\text { Clorofilina cúprica (semi-sintético). } \\
\text { Carotenoides sintéticos. Rojo Ponceau } \\
\text { (azoico). Tartrazina (amarillo, azoico) } \\
\text { Eritrosina (rojo cereza, xanteno) } \\
\text { Amarillo } n^{\circ} 6 \text { (azoico). Rojo } n^{\circ} 1 \\
\text { (amaranto, azoico) } \\
\text { Rojo } 2 \mathrm{G} \text { (rojo azulado, azoico) } \\
\text { Verde } \mathrm{S} \text { (trifenilmetano). } \\
\text { Azul brillante (trifenilmetano) } \\
\text { Azul patente (trifenilmetano) }\end{array}$ \\
\hline & Acidulantes & $\begin{array}{l}\text { Acido fosfórico. Ácido cítrico. Acido tartático. } \\
\text { Sales acidas. }\end{array}$ \\
\hline & $\begin{array}{l}\text { Blanqueantes de } \\
\text { harina de trigo }\end{array}$ & $\begin{array}{l}\text { Peróxido de benzoilo } \\
\text { Ácido perbenzoico } \\
\text { Gases oxidantes: } \mathrm{ClO}_{2}, \mathrm{CINO}\end{array}$ \\
\hline \multirow{2}{*}{$\begin{array}{l}\text { Aditivos que } \\
\text { impiden o retrasan } \\
\text { alteraciones de } \\
\text { los alimentos }\end{array}$} & $\begin{array}{l}\text { Antimicrobiabiónos } \\
\text { (conservadores) }\end{array}$ & $\begin{array}{l}\text { Anhídrido sulfuroso y bisulfitos. } \\
\text { Acido benzoico.Ácido acético. } \\
\text { Ácido propiónico. } \\
\text { Ácido sórbico. } \\
\text { Esteres del ácido p-hidroxí-benzoico. } \\
\text { Nitritos y nitratos. } \\
\text { Antibióticos: nisina, tetraciclinas. }\end{array}$ \\
\hline & $\begin{array}{l}\text { Antioxidantes y } \\
\text { secuestradores de } \\
\text { iones metálicos }\end{array}$ & $\begin{array}{l}\alpha \text {-tocólerol, } \\
\text { Palmitato de ascorbilo y análogos, } \\
\text { Terbutil-hidroxi-anisol (BHA). } \\
\text { DiterbutiU-nielil-Fenol (BHT). } \\
\text { Esteres del acida gálico. } \\
\text { Flavonoides. }\end{array}$ \\
\hline
\end{tabular}




\begin{tabular}{|c|c|c|}
\hline & & $\begin{array}{l}\text { Citratos. } \\
\text { Tartratos. } \\
\text { Ácido trioxigluitárico (TOG) } \\
\text { Acido glucónico. } \\
\text { Polifosfatos. } \\
\text { Acido etilendiamino-tetra-acético. }\end{array}$ \\
\hline \multirow{5}{*}{$\begin{array}{l}\text { Mejorantes de } \\
\text { la textura de los } \\
\text { alimentos }\end{array}$} & $\begin{array}{l}\text { Espesantes y } \\
\text { Gelificantes }\end{array}$ & $\begin{array}{l}\text { Alginatos. } \\
\text { Carragenalos. } \\
\text { Agar. } \\
\text { Gomas de garrofín y de guar. } \\
\text { Celulosos modificadas. } \\
\text { Pectinas. } \\
\text { Almidones. } \\
\text { Almidones modificados. } \\
\text { Gelatina. } \\
\text { Sorbitol y xilitol }\end{array}$ \\
\hline & Emulgentes & $\begin{array}{l}\text { Lecitinas. } \\
\text { Mono y diglicéridos. } \\
\text { Proteínas de leche. } \\
\text { Glicéridos de hidroxiácidos. } \\
\text { Estearato de propilenglicol y análogos. } \\
\text { Esteres de sorbitán. } \\
\text { Esteres de xilitán. } \\
\text { Polioxietilén -éteres de sorbitán. } \\
\text { Poliexietilen-éteres de xilitán. } \\
\text { Esteres de azúcares. }\end{array}$ \\
\hline & Humectantes & $\begin{array}{l}\text { Sorbilol } \\
\text { Xilitol } \\
\text { Isomalt }\end{array}$ \\
\hline & Antiaglomerantes & $\begin{array}{l}\text { Silicatos de } \mathrm{Ca}, \mathrm{Mg} \text {, o Al. Fosfato calcico. } \\
\text { Estearatos de Co y de Mg. }\end{array}$ \\
\hline & $\begin{array}{l}\text { Otros mejorantes de } \\
\text { la textura }\end{array}$ & $\begin{array}{l}\text { Sales solubles de Ca: da firmeza a los } \\
\text { tejidas cuando la pediría está degradada. } \\
\text { Polifosfatos: mejoran la retención de agua y } \\
\text { la consistencia de derivados cárnicos. } \\
\text { Persulfato amónico. } \\
\text { Brómato potásico } \\
\text { Ácido dehidro-ascórbico. } \\
\text { Harina de soja cruda (peroxidasas) }\end{array}$ \\
\hline
\end{tabular}


En definitiva, y como se puede apreciar, en la tabla de clasificación general, dado el elevado número de sustancias aditivas, sería imposible el tratar de estudiar en este curso la totalidad de ellos. Por otra parte, los aditivos tienen propiedades y aplicaciones que están interrelacionadas, por ejemplo, en muchas ocasiones, un colorante es además un potenciador del sabor, o un conservante interviene también en las propiedades que mejoran la textura del alimento, dándole un espesor adecuado. Por estas razones, a continuación, se discutirán algunos aditivos de importancia en alimentos, sin que esto quiera decir que son los únicos empleados.

\section{Tratamientos físicos}

\section{Tratamientos térmicos}

El tratamiento térmico es uno de los procesos más utilizados como método de preparación de los alimentos. La forma en la que el alimento es cocinado (directamente sobre el fuego, hervido, asado, frito, etc.), así como la temperatura y duración del mismo, influyen sobre los cambios químicos y la naturaleza de los productos originados. Debido a este tratamiento en los alimentos se producen una serie de reacciones químicas que dan lugar a derivados más o menos tóxicos que los compuestos de partida pudiendo aparecer distintas sustancias epoxi, hidroxi, peroxi y sustancias cancerígenas. Los procesos más comunes son:

\section{Termooxidación de la fracción lipídica}

Debido a las altas temperaturas, dando lugar al enrranciamiento de los alimentos por formación de monómeros cíclicos, dímeros polímeros, hidroperóxidos y peróxidos.

\section{Formación de compuestos pirorgánicos}

Se forman por las altas temperaturas de carbonización, alrededor de $300^{\circ} \mathrm{C}$ o superiores. Entre ellos destacan: los hidrocarburos policíclicos aromáticos (PAHs), (Figura 9.2) las aminas heterocíclicas y derivados de aminoácidos y la acroleína. Los hidrocarburos policíclicos aromáticos que están formados por de 2 a 4 núcleos aromáticos constituyen uno de los grupos cancerígenos más importantes que pueden afectar a la salud pública. La población puede estar expuesta a ellos a través de humo del tabaco y alimentos contaminados por fuentes 
de combustión o por los procesos de cocinado y tecnológicos a los que se someten. Los alimentos más frecuentemente contaminados son los vegetales, aceites, varios tipos de pescado y carnes asados y ahumados. La incorporación de PAHs a los alimentos es debida principalmente a procesos de cocinado donde su presencia es directamente proporcional a la temperatura de cocinado y al contenido en grasa del alimento. Entre los PAHs más importantes están antracenos, fenantrenos, fluorenos y benzopirenos, siendo el venzo (a) pireno el más tóxico actualmente.

Compuestos derivados de aminoácidos y azúcares

La reacción de Maillard, reacción que tiene lugar entre los aminoácidos y los azúcares reductores en presencia de humedad y calor, sobre todo bajo condiciones alcalinas, puede formar las Melanoidinas y las arcrilamidas.

La acrilamida se forma en los alimentos principalmente por una reacción entre la asparragina y los azúcares reductores. Los mecanismos de formación de estos tóxicos no están del todo definidos, aunque se han señalado, entre otros la reacción entre las grasas, a elevadas temperaturas.

La temperatura de fritura habitual es determinante en la formación de estas sustancias, incrementándose cuanto más se prolonga el tratamiento. Además, los calentamientos sucesivos conllevan un incremento en la concentración. El ejemplo más claro es el del calentamiento en microondas. Así, si después de una fritura intensa se procede a recalentamientos intensos en hornos microondas, las cantidades se ven incrementadas sensiblemente. La formación de acrilamida depende de la presencia de azúcares reductores y algunos aminoácidos. Si se controla el grado de degradación del almidón se podría limitar su acumulación en los alimentos. Sin embargo, esta situación no se pone de manifiesto en alimentos acuosos, como por ejemplo las patatas o las hamburguesas. Ello se debe a la enorme influencia que posee el agua de los alimentos sobre las modificaciones de la temperatura.

Normalmente, en los alimentos con elevada concentración de agua los incrementos de temperatura no son tan importantes. Estos alimentos, siempre que posean una elevada concentración de agua, se mantendrán a $100{ }^{\circ} \mathrm{C}$, la temperatura de cambio de estado del líquido a gas. La concentración de agua puede ser un parámetro determinante en el control de la formación de estas sustancias, siendo los alimentos con elevada humedad relativa los que poseen un menos peligro. En cuanto a los procesos, la temperatura óptima de formación de acrilamida 
parece situarse en torno a los $180^{\circ} \mathrm{C}$, fácilmente alcanzables en muchos de los hornos y sistemas de fritura industriales. Sin embargo, la cocción de patatas fritas u horneadas en casa alcanza con facilidad esa temperatura. En el caso de productos hervidos, la presencia de acrilamida es nula o insignificante (cocción a $100^{\circ} \mathrm{C}$ ). El pan y las galletas son los alimentos con más probabilidades de contener acrilamida, les siguen las patatas fritas, o productos a base de patatas.

Compuestos formados por tratamiento alcalino

Cuando en la industria alimentaria se emplean condiciones alcalinas, no solo se produce la reacción de Maillard, sino que se forman también derivados de aminoácidos con efectos tóxicos como: lisoanalinas, ornitinoalanina y lantionina y aminas (tiramina, triptamina, histamina, cadaverina).

\section{Otras técnicas utilizadas en la industria alimentaria}

Los tratamientos de esterilización y extracción con disolventes pueden dar lugar a residuos no deseados en los alimentos.

\section{CONTAMINACIÓN PRODUCIDA DURANTE ELTRANSPORTE}

En el mundo en que vivimos ha tomado una especial importancia la calidad del transporte alimentario; gracias a él podemos gozar durante todo el año de los alimentos que demandemos. Estos pueden ser producidos en otras partes del mundo, pero a pesar de ello, llegan en inmejorables condiciones a nuestros mercados.

Para conseguir este logro, en Ginebra el 01-09-1970 se creó el Acuerdo sobre transportes internacionales de mercancías perecederas y sobre vehículos especiales utilizados en estos transportes; este acuerdo que es conocido por las siglas ATP, entró en vigor el 21-11-1976, y se actualiza periódicamente. Inicialmente se creó para el transporte internacional y posteriormente el R.D. 1202/ 05 ha hecho que dicho convenio sea también de aplicación a los transportes que se realizan dentro del Territorio Nacional.

Las mercancías de alimentos deberán ser transportadas en vehículos especialmente acondicionados para ello. Dichos vehículos irán identificados individualmente mediante una placa ATP y además en ellos deberá figurar una etiqueta que indique tanto las características del vehículo, como la fecha hasta la que está 
autorizado para dicho tipo de transporte. Aunque existen varias clases, todos comparten en común su baja capacidad de transmisión de calor, impidiendo que la zona donde se transportan los alimentos cambie de temperatura rápidamente debido a las condiciones medioambientales externas.

En los alimentos que requieren mantenerse a bajas temperaturas, la pérdida de la temperatura óptima de refrigeración o congelación en cualquiera de las etapas perjudica la seguridad y la calidad del alimento. Mantener la cadena de frío resulta fundamental a la hora de garantizar la seguridad alimentaria de los alimentos, por lo que todos los eslabones implicados, desde productores hasta distribuidores y detallistas deben poner especial atención en preservarla. La etapa de transporte de alimentos es crucial para mantener la cadena del frío.

\section{CONTAMINACIÓN PRODUCIDA COMO CONSECUENCIA DE LAS OPERACIONES DE MANIPULACIÓN, LIMPIEZA Y DESINFECCIÓN}

En las operaciones de limpieza y desinfección de las superficies, utensilios, cámaras y demás elementos de la industria alimentaria se utilizan diversos productos que pueden dar lugar a contaminaciones si no se eliminan correctamente o se usan productos no adecuados. Entre los productos utilizados se pueden distinguir las lejías, los limpiadores y detergentes y los biocidas ya que mediante la aplicación del sistema de APPCC (Análisis de peligros y puntos críticos de control) todas las empresas tienen obligación de disponer un plan DDD, o lo que es lo mismo un sistema de desinfección, desinsectación y desratización.

\section{Lejías}

Las lejías están reguladas por el RD 3360/83 que obliga a todos los fabricantes, envasadores y comerciantes de lejías para uso doméstico, en colectividades y en la industria alimentaria. Además todos los fabricantes están obligados a comunicar al Instituto Nacional de Toxicología, la composición de las mismas y cualquier cambio que introduzcan, así como de poner en sus productos el etiquetado adecuado.

\section{Detergentes y limpiadores}

Se basan en componentes esenciales llamados agentes tensoactivos y otros 
componentes complementarios. Se incluyen en este grupo productos cuya finalidad es el lavado como el lavado de vajillas, ropa, superficies, etc. Todos regulados por el RD 770/1999.

\section{Biocidas}

El empleo de biocidas está regulado por la Directiva 98/8/CEE relativa a la comercialización de biocidas, y en España por el RD 1054/2002, de 11 de octubre. El objetivo de la norma es controlar los riesgos que los plaguicidas no agrícolas o biocidas pueden implicar para la salud humana, animales y medio ambiente.

Bajo el término de biocida se incluyen los siguientes grupos:

Grupo principal 1: Desinfectantes y biocidas generales.

Grupo principal 2: Conservantes.

Grupo principal 3: Plaguicidas.

Grupo principal 4: Otros biocidas.

En el ámbito de la alimentación humana, localizamos un posible uso entre los desinfectantes y biocidas generales del Grupo principal 1 para los siguientes tipos de productos:

Desinfectantes para las superficies que están en contacto con alimentos

Desinfectantes para agua potable

Por otro lado, los biocidas del Grupo principal 3 se incluyen en plaguicidas para distintos grupos de productos:

Rodenticidas: productos empleados para el control de ratones o ratas $\mathrm{u}$ otros roedores.

Avicidas: productos empleados en el control de aves.

Molusquicidas: productos empleados en el control de moluscos.

Piscicidas: productos empleados para el control de peces.

Insecticidas, acaricidas y productos para el control de otros artrópodos como insectos, arácnidos, crustáceos, etc.

Repelentes y atrayentes: son productos empleados para el control de los organismos nocivos mediante repulsión o atracción, incluidos los empleados directa o indirectamente para la higiene veterinaria o humana.

Los biocidas tienen otros usos relacionados de forma directa o indirecta 
con este ámbito, como son el propio de la alimentación animal (piensos), higiene humana, ámbitos de la vida privada y la salud pública (desinfección del aire, zonas públicas, entre otras) y biocidas para la higiene veterinaria.

\section{BIBLIOGRAFIA}

Pedro Valle Vega, Bernardo Lucas Florentino. Toxicología de los alimentos. Instituto Nacional de Salud Pública. México 2000. ISBN: 9275370044.

MINISTERIO DE AGRICULTURA, PESCA Y ALIMENTACIÓN Subdirección General de Planificación y Control Alimentario. Aromas y aditivos

N. Cubero. A. Monferrer, J. Villalta. Los aditivos alimentarios. Mundi-Prensa Libros, 2002. ISBN: 9788489922785

Ibrahim El, Madfa, Erich Muskat, Doris Fritzsche. Guía de los Aditivos, colorantes y conservantes. Manuales integral. 2003. ISBN: 84-7901-427.X

European Parliament and Council Directive 87/107/EEC (1988) on the approximation of the laws of the Member States concerning Food Aditives authorised for use in foodstuffs intended for human consumption. Official Journal of the European Communities L40, 11.2.89, 27-33.

European Parliament and Council Directive 94/35/EC (1994) on sweeteners for use in foodstuffs. Official Journal of the European Communities L237, 10.9.94, 3-12.

European Parliament and Council Directive 94/36/EC (1994) on colours for use in foodstuffs. Official Journal of the European Communities L237, 10.9.94, 13-29.

European Parliament and Council Directive 95/2/EC (1995) on Food Aditives other than colours or sweeteners. Official Journal of the European Communities L61, 18.3.95, 1-40. 